\title{
Adaptive Modeling Strategy Integrating Feature Selection and Random Forest for Fluid Catalytic Cracking Processes
}

\author{
Chen Chen, Li Zhou, ${ }^{*}$ Xu Ji, Ge He, Yiyang Dai, and Yagu Dang \\ Department of Chemical Engineering, Sichuan University, Chengdu 610065, P.R. China. \\ E-mail: chezli@scu.edu.cn \\ Phone: +86 15228867167
}

\section{Detailed introduction to the RF modeling technique}

RF is an algorithm based on tree predictors proposed by Breiman and Cutler in $2001 .^{\text {It }}$ It uses the bootstrap resampling method to extract multiple samples from original samples and conduct decision tree modeling for each bootstrap sample, and then combine the prediction of multiple decision trees to obtain the final prediction result through voting. Compared with traditional machine learning methods such as artificial neural network, RF is faster to train and has fewer parameters. It also has high prediction accuracy, good tolerance to outliers and noises, and less overfitting. During the training, RF can identify the important degree of the input features, ${ }^{2}$ to some extent, this can enhance feature selection. The schematic diagram of RF is shown in Figure S1. 


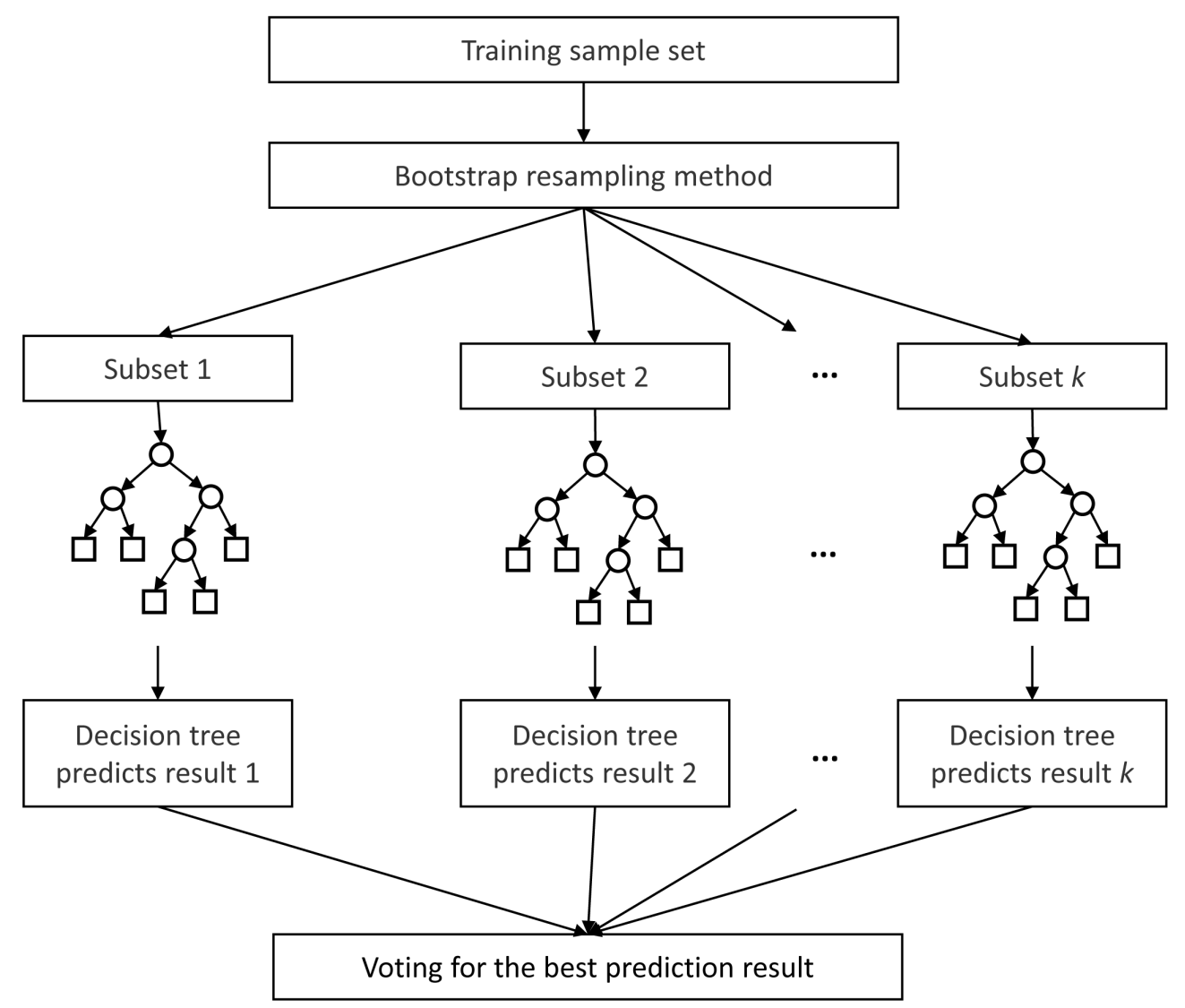

Figure S1: The schematic diagram of random forest. 


\section{The calculation of the "antibody" concentration, adaptive crossover and mutation probability}

\section{The calculation of the antibody concentration.}

Similar to the biological immune system, AIGA promotes the high affinity and low concentration of antibodies, on the contrary, inhibits the high concentration and low affinity of antibodies. The concentration represents the number of similar antibodies scale, which is calculated by the average information entropy. For an antibody group that has $N$ antibodies and each antibody has $m$ gene sites which has optional $z$ symbols, antibodies' average information entropy $H(N)$ is defined as Equation $1.3^{3}$

$$
H(N)=\frac{1}{m} \sum_{i=1}^{m} H_{i}(N)
$$

where $H_{i}(N)$ denotes the information entropy of the $i$ th gene:

$$
H_{i}(N)=\sum_{j=1}^{S}-P_{i j} \ln P_{i j}
$$

the $P_{i j}$ is the probability of a particular symbol in $z$ symbols (in this study, 0 or 1 ) of the $i$ th gene of the antibody. The similarity between $v$ th antibody and wth antibody is explained by Equation 3.

$$
S_{v, w}=\frac{1}{1+H(2)}
$$

subject to

$$
S_{v, w} \in[0,1]
$$

$H(2)$ is the information entropy between two antibodies. When $H(2)$ equals to zero, then $S_{v, w}=1$. That is to say, all genes of the two antibodies are identical. Equation 4 shows the 
concentration of the $v$ th antibody.

$$
C_{v}=\frac{1}{N} \sum_{w=1}^{N} S_{v, w}
$$

AIGA evaluates antibodies by reproductive probability $\left(p_{v}\right)$ and produces the next generation through roulette wheel..$^{-4}$ The $p_{v}$ of $v$ th antibody is controlled by the affinity and the concentration, which is proportional to affinity and inversely proportional to concentration. $p_{v}$ is described as Equation 5.

$$
p_{v}=\sqrt{\frac{r A_{v}}{\sum_{w=1}^{N} A_{w}} / \frac{(1-r) C_{v}}{\sum_{w=1}^{N} C_{w}}}
$$

where $A_{v}$ represents the affinity of $v$ th antibody and $A_{w}$ represents the affinity of $w$ th antibody. $r$ is the diversity evaluation parameter with values from 0 to 1 .

Moreover, to avoid elimination of antibodies with the highest affinity value due to their high concentration, $R$ individuals with the highest affinity value in the antibodies will be stored in the memory unit, and the remaining $N-R$ individuals will enter the immune selection.

\section{Adaptive crossover and mutation.}

In this study, single-point crossover $^{5}$ is operated between two cross parents which selected randomly to create their offspring according to the crossover probability $\left(p_{c}\right)$. And the simple mutation ${ }^{6}$ performs random perturbations to the selected antibodies based on the mutation probability $\left(p_{m}\right) . p_{c}$ and $p_{m}$ are two crucial parameters in AIGA, which determine the performance of the algorithm to a large extent. To further maintain antibody diversity, AIGA adopts average information entropy to calculate the similarity of the whole antibodies $S(N)$ :

$$
S(N)=\frac{1}{1+H(N)}
$$


subject to

$$
S(N) \in[0,1]
$$

Then, AIGA adjusts crossover and mutation probability adaptively according to the similarity of antibodies. When the affinities of antibodies tend to be consistent or the local optimal, $p_{c}$ and $p_{m}$ increase. On the other hand, when the overall affinity value is discrete, $p_{c}$ and $p_{m}$ decrease. $p_{c}$ and $p_{m}$ are adjusted adaptively according to the following formula: $:^{7}$

$$
\begin{gathered}
p_{c}=\exp [2(S(N)-1)] \\
p_{m}=0.1 \exp [2(S(N)-1)]
\end{gathered}
$$

\section{AIGA-RF model parameters setting}

In this case study, the AIGA-RF model parameters settings are shown in Table S1.

Table S1: AIGA-RF Model Parameters Setting.

\begin{tabular}{ccc}
\hline parameter & meaning & value \\
\hline$m$ & the number of all the collected process features & 240 \\
$N$ & the number of initialized antibodies & 100 \\
$R$ & The capacity of the & 10 \\
$r$ & recorded high affinity antibodies & 0.95 \\
$n$ & the diversity evaluation parameter & 10 \\
max_features & the number of decision trees in the RF & 240 \\
& the number of features to consider & \\
min_samples_leaf & the minimum number of samples & 1 \\
& in newly created leaves of a single decision tree & \\
\hline
\end{tabular}

\section{References}

(1) Breiman, L. Random Forests. Machine Learning 2001, 45, 5-32.

(2) Cano, G.; Garcia-Rodriguez, J.; Garcia-Garcia, A.; Perez-Sanchez, H.; Benedikts- 
son, J. A.; Thapa, A.; Barr, A. Automatic selection of molecular descriptors using random forest: Application to drug discovery. Expert Systems with Applications 2017, 72, $151-159$.

(3) Liu, X.; Yang, Z.; Pan, W. An improved adaptive immune genetic algorithm based on information entropy. 2015 International Conference on Industrial Informatics - Computing Technology, Intelligent Technology, Industrial Information Integration. 2015; pp 6-9.

(4) Zames, G.; Ajlouni, N.; Ajlouni, N.; Ajlouni, N.; Holland, J.; Hills, W.; Goldberg, D. Genetic algorithms in search, optimization and machine learning. Information Technology Journal 1981, 3, 301-302.

(5) Li, J.; Sang, H.; Han, Y.; Wang, C.; Gao, K. Efficient multi-objective optimization algorithm for hybrid flow shop scheduling problems with setup energy consumptions. Journal of Cleaner Production 2018, 181, 584-598.

(6) Zhang, Y.; Gong, Y.; Gu, T.; Li, Y.; Zhang, J. Flexible genetic algorithm: A simple and generic approach to node placement problems. Applied Soft Computing 2017, 52, $457-470$.

(7) Que, Y.; Zhong, W.; Chen, H.; Chen, X.; Ji, X. Improved adaptive immune genetic algorithm for optimal QoS-aware service composition selection in cloud manufacturing. The International Journal of Advanced Manufacturing Technology 2018, 96, 4455-4465. 